\title{
Molecular Characterization of gyrA, parC and qepA Genes in Quinolone Resistant ESBL Producing E. Coli Isolated from Patients in HTAA, Kuantan
}

Rahmatullah Sirat ${ }^{1}$, Hairul Aini Hamzah² ${ }^{2}$ Mohammed Imad A. Mustafa Mahmud ${ }^{1}$, Siti Nurliyana Binti Ahmad ${ }^{3}$

${ }^{1}$ Department of Basic Medical Sciences, International Islamic University Malaysia

${ }^{2}$ Department of Para-clinic,Medicine Faculty, Kandahar University, Afghanistan

${ }^{3}$ Department of Pathology, Mircrobiology Unit, Hospital Tengku Ampuan Afzan, Kuantan, Pahang

Presenter: Rahmatullah Sirat

Introduction: Quinolone resistance and extended spectrum beta lactamase production has increased in E.coli and considered a serious problem worldwide. It is worth to monitor resistance mechanism in E.coli to provide guidance for optimizing antimicrobial treatments, control and spread of resistance. The objective of this study was to molecularly characterize gyrA, parC genes and plasmid mediated qepA efflux pump gene, in QR-ESBL $E$. coli isolates obtained from patients in HTAA, Kuantan. The antibiotic susceptibility profile was also studied. Materials and Method: 32 QR-ESBL and six quinolone-susceptible E. coli isolates from September 30 November,2018) included in the study. The isolates were reconfirmed with known phenotypic tests and antibiotic susceptibility test was performed. PCR and DNA sequencing were performed for the identification of mutations in quinolone resistance determining region. Result: Resistance to ampicillin, tetracycline, nalidixic acid was (100\%) followed by cefotaxime $(96.9 \%)$, ciprofloxacin (78.1\%) trimethoprim sulfamethoxazole (75\%), ceftazidime $(56.3 \%)$, cefepime (43.8\%) and gentamycin (25\%). None of the isolates was resistant to piperacillin-tazobactam, amikacin, imipenem, meropenem, ertapenem, and colistin. PCR successfully amplified the gyrA and parC genes, however, qepA gene was not detected by PCR in the isolates. Majority of the isolates had point mutation in (QRDR) of GyrA at codons 83 and 87 and in ParC at codons 80 and 84. Two isolates had mutations outside of QRDR at codons 144 and 167 in ParC. Strong positive correlation was found between MIC levels of ciprofloxacin and the number of resistance mutations. Sequencing of 6 (QS-ESBL) E. coli revealed absence of resistance mutations. Conclusion: Quinolone resistance in the isolates was mainly due to mutations in gyrA, ParC genes. Acquisition of multidrug resistance genes through innate gene mutations and mobile genetic elements contribute to the emergence of (MDR). This study reinforces the importance of being vigilant in utilizing molecular techniques to monitor for emergence of resistance genes in different locations. 